\begin{tabular}{|c|l|}
\hline Title & Fluctuating superconductivity in kappa type organic compounds probed by polarized time resolved spectroscopy \\
\hline Author(s) & Nakagawa, Koichi; Tsuchiya, Satoshi; Y amada, Jun-ichi; Toda, Y asunori \\
\hline Citation & $\begin{array}{l}\text { EPL, 122(6), } 67003 \\
\text { https://doi.org/40.1209/0295-5075/L22/67003 }\end{array}$ \\
\hline Issue Date & 2018-06 \\
\hline Doc URL & http://hdl.handle.net/2115/74508 \\
\hline Type & article(author version) \\
\hline File Information & Text_manuscript(revised) -huscap.pdf \\
\hline
\end{tabular}

Instructions for use 


\title{
Fluctuating superconductivity in $\kappa$-type organic compounds probed by polarized time-resolved spectroscopy
}

\author{
Koichi Nakagawa ${ }^{1}$, Satoshi TsuchiYa ${ }^{1}$, Jun-ichi Yamada $^{2}$ and Yasunori Toda ${ }^{1}$ \\ 1 Graduate School of Engineering, Hokkaido University - Kita13 Nishi8 Kitaku, Sapporo, Hokkaido 060-8628, Japan \\ 2 Graduate School of Material Science, University of Hyogo - 3-2-1 Kouto, Kamigori-cho, Ako-gun, Hyogo 678-1297, \\ Japan
}

PACS 74.70.Kn - Organic superconductors

PACS 78.47.jg - Time resolved reflection spectroscopy

\begin{abstract}
In this work, polarized time-resolved spectroscopy was carried out to investigate a superconducting (SC) state of the organic compounds $\kappa$-(BEDT-TTF $)_{2} X\left(X=\mathrm{Cu}(\mathrm{NCS})_{2}(\kappa-\mathrm{NCS}\right.$, $\left.T_{\mathrm{c}} \sim 9 \mathrm{~K}\right)$ and $\mathrm{Cu}\left[\mathrm{N}(\mathrm{CN})_{2}\right] \mathrm{Br}\left(\kappa\right.$-Br, $\left.\left.T_{\mathrm{c}} \sim 12 \mathrm{~K}\right)\right)$, which have different effective electron correlations. We found that the $\mathrm{SC}$ response, which was characterized by probe polarization anisotropy with slow relaxation time, decreases steeply at approximately $T_{\mathrm{c}}$ in $\kappa$-NCS with increasing temperature, corresponding to the closing of the SC gap. On the other hand, in $\kappa$ - $\mathrm{Br}$ the SC response persisted significantly above $T_{\mathrm{c}}$. The results indicate that fluctuating superconductivity (FSC) emerges in $\kappa$-Br but not in $\kappa$-NCS, suggesting that strong electron correlation can play a critical role for FSC.
\end{abstract}

Introduction. - In conventional superconductors, as the temperature increases across the transition temperature $\left(T_{\mathrm{c}}\right)$, the amplitude of the order parameter $|\Psi|$ corresponding to the density of the Cooper pairs vanishes, and simultaneously bulk phase coherence is lost. On the other hand, strongly correlated layered superconductors such as cuprates and organics can exhibit fluctuating superconductivity (FSC), which is characterized by a finite $|\Psi|$ value without bulk phase coherence above $T_{\mathrm{c}}$. This type of FSC is distinguished from the Aslamazov-Larkin and Maki-Thompson superconducting fluctuation. Indeed, Nernst effect measurements in the cuprates have suggested FSC [1,2]. However, a pseudogap (PG) formation above $T_{\mathrm{c}}[3]$, in which recent studies have suggested density wave transitions $[4,5]$ and inhomogeneous superconductivity, reportedly related to the quenched disorder $[6,7]$, makes it difficult to investigate FSC further.

A series of superconductors $\kappa$-(BEDT-TTF $)_{2} X$ (X: anion molecules) is suitable candidate for understanding the mechanism of FSC because any density wave states or quenched disorder have not been reported in spite of the similarity with the cuprates in regard to their electronic properties. The recent systematic Nernst effect measurements $[8,9]$ have suggested that FSC appears at $18 \mathrm{~K}$ in $\kappa$-(BEDT-TTF $)_{2} \mathrm{Cu}\left[\mathrm{N}(\mathrm{CN})_{2}\right] \mathrm{Br}[10]$ (hereafter $\kappa$-Br) with $T_{\mathrm{c}}$ of $12 \mathrm{~K}$ but not in $\kappa$-(BEDT-TTF $)_{2} \mathrm{Cu}(\mathrm{NCS})_{2}[11](\kappa-$
NCS) with $T_{\mathrm{c}}$ of $9 \mathrm{~K}$, where $T_{\mathrm{c}} \mathrm{S}$ are cited from the specific heat measurements [12]. On the other hand, magnetic torque measurements $[13,14]$ have suggested the presence of FSC in $\kappa$ - $\mathrm{Br}$ as well as in $\kappa$-NCS although magnetic susceptibility measurements [15] have consistently agreed with the Nernst effect measurements. In addition, ${ }^{13} \mathrm{C}$ NMR measurements [16] have indicated that the FSC effect appears only in $\kappa$ - $\mathrm{Br}$ at $13-14 \mathrm{~K}$, which is quite low compared to the Nernst effect measurements. In this way, FSC in the organic system is still controversial.

To obtain convincing evidence of FSC, spectroscopic measurements can be valid because they can provide information on the SC gap in the electronic energy spectrum. In particular, optical pump-probe spectroscopy using near-infrared and ultraviolet light pulses [17-19] is one of the most reliable techniques for an investigation of FSC. In the measurements, photoinduced carrier relaxation dynamics of FSC are detectable as well as the bulk SC state because the presence of an energy gap is reflected efficiently in the dynamics. Moreover, even if the dynamics originating in different states, such as the $\mathrm{SC}$ or PG states, overlap in a coexistence state, they can be separated by differences in probe polarization anisotropy, relaxation time, temperature dependences, and so on.

In this paper, we report the photoinduced carrier relaxation dynamics in the SC state of $\kappa$-Br and $\kappa$-NCS, 
which are characterized by probe polarization anisotropy with slow relaxation time. When the temperature increases across $T_{\mathrm{c}}$, the component vanishes steeply at approximately $T_{\mathrm{c}}$ in $\kappa$-NCS, corresponding to the closing of the superconducting gap, whereas it reduces gradually and persists significantly above $T_{\mathrm{c}}$ in $\kappa$ - $\mathrm{Br}$. The result indicates that the FSC emerges in $\kappa$-Br but not in $\kappa$-NCS. The qualitative difference between the salts is consistent with the previous Nernst effect and the magnetic susceptibility measurements, indicating that strong electron correlation can play a crucial role for FSC.

Optical measurements. - Single crystals of $\kappa$-NCS and $\kappa$-Br were prepared electrochemically [20]. The crystals consist of alternately stacked BEDT-TTF conducting layers and insulating anion layers, forming a quasitwo-dimensional electronic structure. As shown in fig. 1(a), dimerization of BEDT-TTF molecules provides a half-filled energy band. The application of pressure corresponds to an increase in the effective electron correlation $t / U$, where $t$ and $U$ denote the transfer integral and the onsite Coulomb repulsion, respectively. It has been pointed out that $\kappa$-Br has a smaller $t / U$ value than $\kappa$-NCS does, indicating that its electronic state is located closer to the Mott phase than that of $\kappa$-NCS, as shown in a phase diagram fig. 1 (b).

In the optical measurements, a cavity-dumped Ti:sapphire oscillator was used to obtain the pump and probe beam pulses, which have a duration of $\sim 120$ fs with a repetition rate of $54 \mathrm{kHz}$ to suppress the heating effect. The pump and probe beams centered at $400 \mathrm{~nm}$ $(3.1 \mathrm{eV})$ and $800 \mathrm{~nm}(1.55 \mathrm{eV})$, respectively, were coaxially overlapped and irradiated perpendicular to the conducting planes. The probe polarization was rotated by a half-wave plate and its angle $\theta_{\mathrm{pr}}=0^{\circ}$ corresponded to the direction tilted by $45^{\circ}$ from the $b(c)$ axis for $\kappa$-NCS $(\kappa$ $\mathrm{Br}$ ), which is inferred from the previous report [21]. The measurements were carried out with pump fluence values $\mathcal{F}=19 \mu \mathrm{J} / \mathrm{cm}^{2}$ for $\kappa$-NCS and $13 \mu \mathrm{J} / \mathrm{cm}^{2}$ for $\kappa$-Br. We carefully cooled the samples at a rate of $0.1-1.0 \mathrm{~K} / \mathrm{min}$ to avoid disordering the terminal ethylene groups of the BEDT-TTF molecules [22].

In the pump-probe measurements, the pump pulse excites carriers to a non-equilibrium high energy state. The excited carriers immediately relax to a state near the Fermi level via electron-electron and electron-phonon scatterings. The relaxation process is observed by delayed probe pulses as a change in reflectivity $\Delta R\left(\theta_{\mathrm{pr}}\right) / R$. When an SC gap is present, a relaxation bottleneck effect [23] may occur and the carriers accumulate just above the gap, leading to a drastic amplitude change in $\Delta R / R$ and decay time at $T_{\mathrm{c}}$. It is important to note that the relaxation dynamics can also reflect FSC above $T_{\mathrm{c}}$ because the Cooper pair density remains without bulk phase coherence [24].
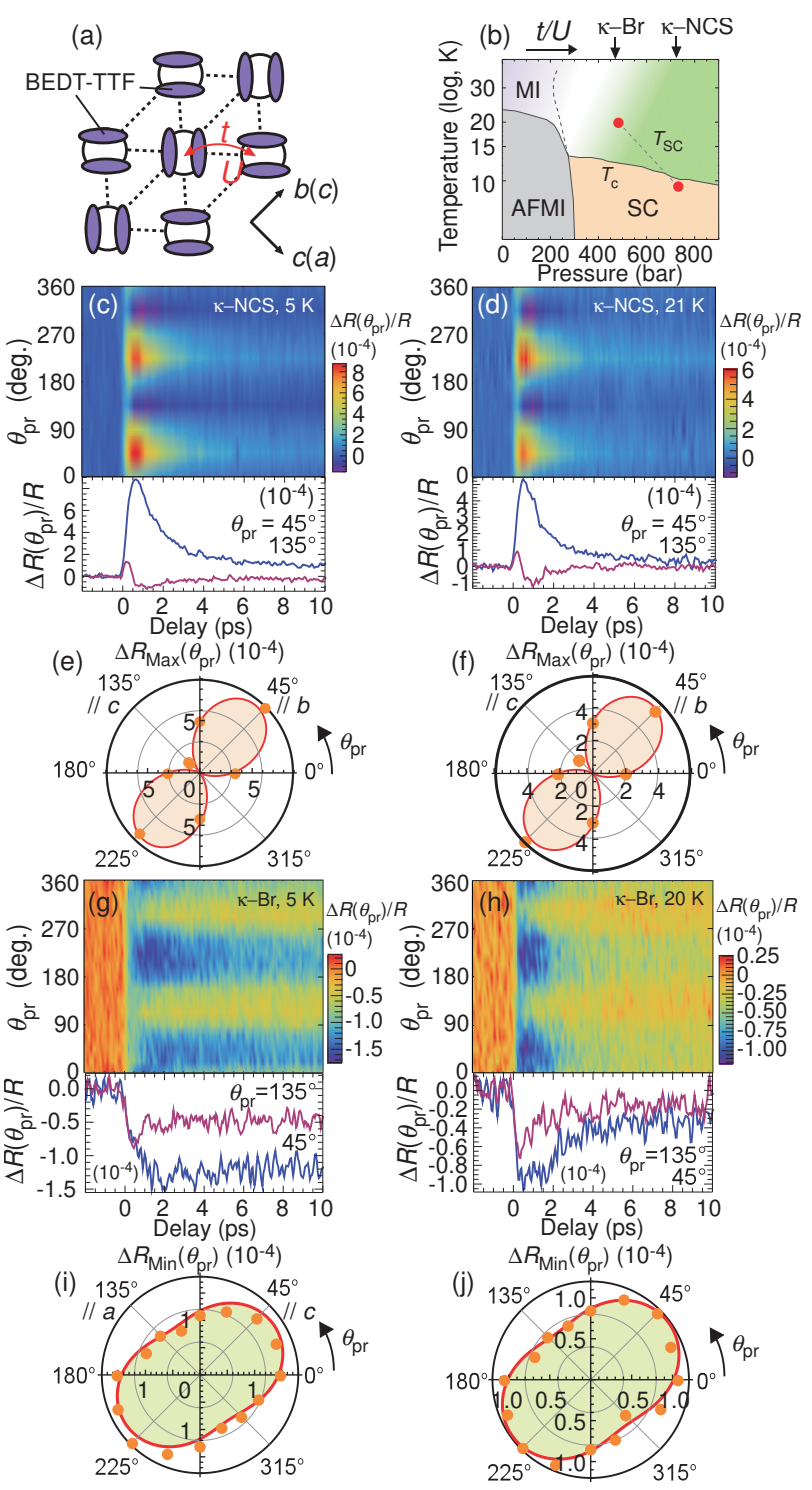

Fig. 1: (Color online) (a) Schematic of BEDT-TTF molecular arrangement of a conducting layer. (b) Temperaturepressure phase diagram of electronic states in $\kappa$-(BEDT$\mathrm{TTF})_{2} X$. AFMI, MI, and SC represent antiferromagnetic Mott insulating, Mott insulating and superconducting phases, respectively. (c) and (d) Density plots of $\Delta R / R$ transients as a function of $\theta_{\mathrm{pr}}$ and $\Delta R / R$ transients for $\theta_{\mathrm{pr}}=45^{\circ}$ and $135^{\circ}$ in $\kappa$-NCS at $T=5 \mathrm{~K}$ and $21 \mathrm{~K}$, respectively. (e) and (f) Polar plots of $\theta_{\mathrm{pr}}$ dependence of maximum values $\Delta R_{\mathrm{Max}}$. (g) and (h) Density plots of $\Delta R / R$ transients as a function of $\theta_{\mathrm{pr}}$ and $\Delta R / R$ transients for $\theta_{\mathrm{pr}}=45^{\circ}$ and $135^{\circ}$ in $\kappa$ - $\mathrm{Br}$ at $T=5 \mathrm{~K}$ and $20 \mathrm{~K}$, respectively.

(i) and (j) Polar plots of $\theta_{\text {pr }}$ dependence of minimum values $\Delta R_{\mathrm{Max}}$. The solid lines are the results fitted by eq. (1). 
Polarization dependence analysis. - Figures $1(\mathrm{c})-(\mathrm{d})$ and $(\mathrm{g})-(\mathrm{h})$ show density plots of $\Delta R\left(\theta_{\mathrm{pr}}\right) / R$ transients as a function of $\theta_{\mathrm{pr}}$ at $5 \mathrm{~K}$ and $21 \mathrm{~K}$ in $\kappa$-NCS and at $5 \mathrm{~K}$ and $20 \mathrm{~K}$ in $\kappa$-Br, respectively. The bottom panels show $\Delta R\left(\theta_{\mathrm{pr}}\right) / R$ for $\theta_{\mathrm{pr}}=45^{\circ}$ and $135^{\circ}$. In figs. 1 (e)-(f) and (i)-(j), the probe polarization angular dependences of the signal amplitude at below and above $T_{\mathrm{c}}$ are shown as the polar plots for $\kappa$ - NCS and $\kappa$-Br, respectively. The signals are enhanced along the direction of the $b$ axis for $\kappa$-NCS and the $c$ axis for $\kappa$-Br even above $T_{\mathrm{c}}$. The appearance of the anisotropy is due to the spatially anisotropic change of the optical transition probability. The detail process has been reported as the photoinduced phase separation [21] owing to the pump induced destruction of the local ordering of the BEDT-TTF molecular conformation emerging below the glass-like transition temperature temperature [22]. When the temperature decreases below $T_{\mathrm{c}}$, the directions of the anisotropy are not changed, but the amplitude of the signals increases in both the compounds. This indicates that the cosine symmetry in the SC state has quite similar to the anisotropy emerging at the glass-like transition temperature in contrast to the optically orthogonal relation between the pseudogap and SC symmetry in Bi2212 [25].

For further analysis, we decomposed the data into anisotropic and isotropic components by using fits with the following relation:

$$
\Delta R_{\mathrm{Max} / \mathrm{Min}}\left(\theta_{\mathrm{pr}}\right)=\Delta R_{\mathrm{ani}} \cos \left\{2\left(\theta_{\mathrm{pr}}-\phi_{\mathrm{c}}\right)\right\}+\Delta R_{\text {iso }},
$$

where $\Delta R_{\text {ani }}, \Delta R_{\text {iso }}$ and $\phi_{\mathrm{c}}$ denote the amplitudes of anisotropic and isotropic components and a phase, respectively. $\phi_{\mathrm{c}}$ has an almost constant value of approximately $\sim 45^{\circ}$ for the temperature variation within the errors. Figures 2 (a) and (c) show the density plots of $\Delta R_{\text {ani }}$ with the insets for those of $\Delta R_{\text {iso }}$. In both the salts, $\Delta R_{\text {iso }}$ develops at low temperature around $T_{\mathrm{c}}$, which is quite similar to the behavior of $\Delta R_{\text {ani }}$. In principle, both the isotropic and anisotropic channels are sensitive to the SC component [25]. Note that the isotopic channel is sensitive to any kinds of the relaxation components including the metallic, the $\mathrm{SC}$, or the other components, reflecting spectral change induced by the pump pluses. On the other hand, the anisotropic channel can exclude the metallic component, resulting in the effective detection of the SC dynamics since it extracts the optically anisotropic transition process such as symmetrical change in the electronic ordered states [26] or the anisotropic change in the molecular structural ordered states. Thus, we concentrate on the anisotropic component $\Delta R_{\text {ani }}$ in the following analysis to achieve the selective detection of the SC dynamics.

Characterization of anisotropic signal. - We now focus on $\Delta R_{\text {ani }}$ in $\kappa$-NCS and $\kappa$-Br shown in figs. 2 (a) and (c), respectively. In both the salts, $\Delta R_{\text {ani }}$ is enhanced below around $T_{\mathrm{c}}$. Figures $2(\mathrm{~b})$ and (d) show logarithmic plots of $\Delta R_{\text {ani }}$ at selected temperatures in $\kappa$-NCS and $\kappa$ -
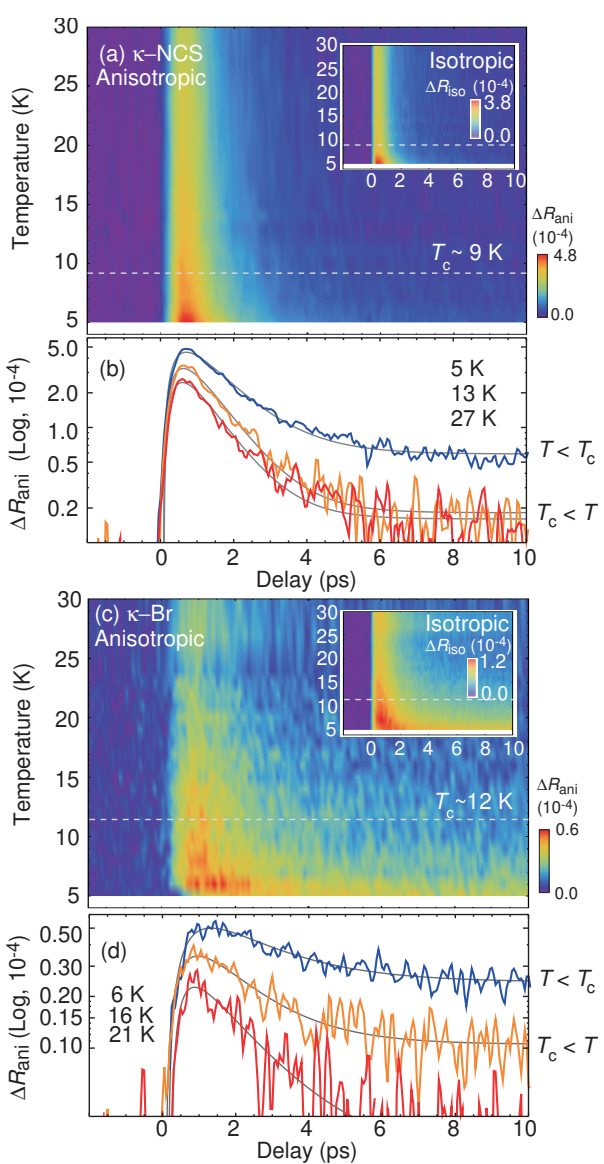

Fig. 2: (Color online) (a) and (c) Density plot of $\Delta R_{\text {ani }} / R$ as a function of temperature in $\kappa$-NCS and $\kappa$ - $\mathrm{Br}$, respectively. The insets indicate the density plot of $\Delta R_{\text {iso }} / R$ as a function of temperature in $\kappa$-NCS and $\kappa$-Br, respectively. (b) and (d) Logarithmic plot of $\Delta R_{\text {ani }} / R$ at selected temperature in $\kappa$-NCS and $\kappa$-Br, respectively.

$\mathrm{Br}$, respectively. In both the salts, we can see $\Delta R_{\text {ani }}$ consists of multiple decay components, where the long-lived component becomes dominant at the lower temperature. However, in $\kappa$-NCS, the component is significantly suppressed at $13 \mathrm{~K}$ while in $\kappa$ - $\mathrm{Br}$, it still remains even at 16 $\mathrm{K}$.

By assuming infinite decay time of the long-lived decay component, the data were fitted with the form $A_{\text {fast }} \exp (-t / \tau)+A_{\text {slow }}$, where $A_{\text {fast }}$ and $A_{\text {slow }}$ represent the amplitudes of the fast decay and long-lived components and $\tau$ is the decay time of the fast component.

Figures 3 (a) and (b) show the temperature dependence of $A_{\text {slow }}$ (circles) in $\kappa$-NCS and $\kappa$-Br, respectively. To show the reproducibility of the data, we also plotted the data obtained under saturation condition of the SC state, as shown by squares. The saturation threshold is estimated to be around $20-50 \mu \mathrm{J} / \mathrm{cm}^{2}$ for both the salts [27]. Here, one of the two data in each figure is multiplied by appropriate factor for better comparison. Their insets show the temperature dependence of $A_{\text {fast }}$ (circles). In both com- 


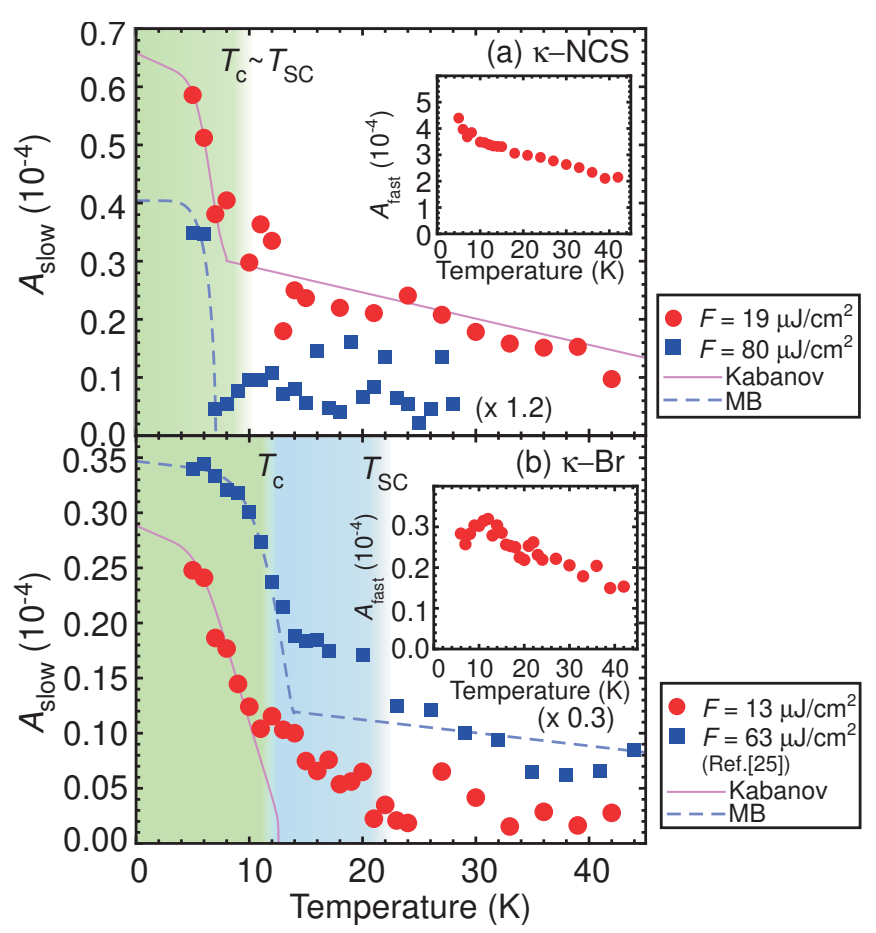

Fig. 3: (Color online) (a) and (b) Temperature dependence of $A_{\text {slow }}$ under $\mathcal{F}=19 \mu \mathrm{J} / \mathrm{cm}^{2}$ (circles) and $80 \mu \mathrm{J} / \mathrm{cm}^{2}$ (squares) in $\kappa$-NCS and $\mathcal{F}=13 \mu \mathrm{J} / \mathrm{cm}^{2}$ (circles) and 63 $\mu \mathrm{J} / \mathrm{cm}^{2}$ (squares) in $\kappa$-Br, respectively. The solid and dashed lines display the fitted curve by eqs. (2) and (3), respectively. The insets correspond to the temperature dependence of $A_{\text {fast }}$ under the lower fluence.

pounds, $A_{\text {fast }}$ slightly increases below $\sim 40 \mathrm{~K}$ and shows drastic changes below $T_{\mathrm{c}}$. The behaviors are consistent with our previous study.

As for $A_{\text {slow }}$ in $\kappa$-NCS, it shows the linear increase with decreasing temperature and the steep development below the onset temperature $T_{\mathrm{SC}} \sim 9 \mathrm{~K}$, which is highly comparable with $T_{\mathrm{c}}$. The steep change corresponds to the bulk-SC transition. To show the SC transition clearly, we plotted $A_{\text {slow }}$ under the higher pump excitation $\mathcal{F}=80$ $\mu \mathrm{J} / \mathrm{cm}^{2}$ (squares) in fig. 3 (a). In this case, $A_{\text {slow }}$ is completely suppressed at the higher temperature but abruptly develops at $7 \mathrm{~K}$. The slight difference of the onset temperature may be due to the temperature gradient between a thermometer and the sample in the cryostat, or the sample quality.

In contrast, $A_{\text {slow }}$ in $\kappa$-Br under the low excitation (circles) starts to grow at $T_{\mathrm{SC}} \sim 20 \mathrm{~K}$ and shows the gradual development below around $T_{\mathrm{c}}$ (i.e. bulk-SC transition). Under the higher excitation $\mathcal{F}=63 \mu \mathrm{J} / \mathrm{cm}^{2}$ (squares), though $A_{\text {slow }}$ shows a linear increase down to around $T_{\mathrm{SC}} \sim 20 \mathrm{~K}$ with lowering temperature, it shows the deviation at $T_{\mathrm{SC}}$ from the linear dependence and the steep increase below around $T_{\mathrm{c}}$. As is discussed in the following section, the gradual change in the temperature range between $T_{\mathrm{c}}$ and $T_{\mathrm{SC}}$ is attributed to FSC.
The linearly temperature-dependent component was observed over the whole temperature range in $\kappa$-NCS under $\mathcal{F}=19 \mu \mathrm{J} / \mathrm{cm}^{2}$ and in $\kappa$-Br under $63 \mu \mathrm{J} / \mathrm{cm}^{2}$. At present, we cannot identify the origin of this component. However, the component is independent of the fluence value, thus, it may be contributed by the thermal effect depending on the sample surface. In other words, the efficiency of the energy diffusion from the hot spot to the environment may be inhomogeneous on the sample surface due to the low heat conduction of the organics. If the component is removed from $A_{\text {slow }}$, the temperature dependences in each fluence are nearly identical.

Comparison with theoretical models. - For reproduction of the SC gap formation under the weak excitation, a fit was carried out with the Kabanov's formula [23]:

$$
\begin{aligned}
A_{\text {slow }}(T) & \propto\left[\Delta_{\mathrm{SC}}(T)+\frac{k_{B} T}{2}\right]^{-1} \\
& \times\left\{1+g \sqrt{\frac{k_{B} T}{\Delta_{\mathrm{SC}(\mathrm{T})}}} \exp \left[-\frac{\Delta_{\mathrm{SC}(\mathrm{T})}}{k_{B} T}\right]\right\}^{-1},
\end{aligned}
$$

where $g$ corresponds to the effective electronic and phononic density of states participating in the relaxation process and $k_{B}$ is the Boltzmann constant. Here, we assume the linearly temperature-dependent component over the whole temperature range for $A_{\text {slow }}$ in $\kappa$-NCS under the low excitation (and $\kappa$-Br under the high excitation). As shown by the solid lines in fig. 3, the data were well fitted below around the $T_{\mathrm{c}}$ of $9 \mathrm{~K}$ in $\kappa$-NCS and $12 \mathrm{~K}$ in $\kappa$-Br, indicating that the developments are attributed to the bulk SC. The fit yields $\Delta_{\mathrm{SC}}(0) \sim 3 \mathrm{meV}$ for both the samples, which is smaller than those in the previous studies $[16,28-30]$. The discrepancy is reasonable because the SC state in these compounds should be characterized by an anisotropic gap, which does not follow the BCS theory used in the model. Under the higher excitation conditions corresponding to the saturation condition of the transient signals, the reproduction was carried out with the MattisBardeen (MB) model [31,32]:

$$
A_{\text {slow }}(T) \propto \Delta_{\mathrm{SC}}(T)^{2} \log \left[\frac{\hbar \omega}{\Delta_{\mathrm{SC}}(T)}\right],
$$

where $\hbar \omega$ and $\Delta_{\mathrm{SC}}(T)$ are the probe photon energy and a BCS-type gap function, respectively. The dashed curve in fig. 3 (a) reproduces the steep change at $\sim 7 \mathrm{~K}$ in $\kappa$-NCS and yields $\Delta_{\mathrm{SC}}(0) \sim 1 \mathrm{meV}$, which is smaller than the values obtained from other measurements. We note that the onset temperature and $\Delta_{\mathrm{SC}}$ may contain certain error due to the experimental condition and the incompleteness of the theoretical model. For $\kappa$ - Br in fig. 3 (b), the linearly temperature-dependent component is also assumed in the whole temperature range for the high excitation condition and $A_{\text {slow }}$ below $T_{\mathrm{SC}}$ is well described by the dashed curve 
with the estimation of $\Delta_{\mathrm{SC}} \sim 7 \mathrm{meV}$.

Comparison with other measurements. - Here, we discuss the origin of the slow decay component above $T_{\mathrm{c}}$ in $\kappa$ - $\mathrm{Br}$ in comparison with the other previous measurements. In the Nernst measurements [8], the signal appears clearly in $\kappa$-Br at $18 \mathrm{~K}$, which is significantly above $T_{\mathrm{c}}$, while the signal appears at just $T_{\mathrm{c}}$ in $\kappa$-NCS. The enhancement of the Nernst signal in $\kappa$ - $\mathrm{Br}$ can be interpreted as the emergence of FSC. In the FSC state, thermally excited vortices and anti-vortices flow, destroying the ordering of the superconducting phase. In the Nernst measurements, when a magnetic field is applied and the temperature gradient is induced perpendicular to the field in the sample, vortices can flow along the temperature gradient, resulting in an electrical voltage perpendicular to the temperature gradient and field, which is called the vortex-Nernst signal. In our results, as plotted by red circles in fig. 1 (b), $T_{\mathrm{SC}}$ increases with decreasing $t / U$. Because this is in excellent agreement with the results of the Nernst effect measurements, the slow decay component may originate in the comparable FSC state.

Moreover, in the magnetic susceptibility measurements with an SC quantum interference device (SQUID), by measuring the difference in magnetic susceptibility under magnetic fields parallel and perpendicular to the conducting layers, the diamagnetism due to FSC has been successfully distinguished from the normal state susceptibility in $\kappa$ - $\mathrm{Br}$ [15]. As a result, the onset temperature of diamagnetism is suggested to be $\sim 19 \mathrm{~K}$, which is also very close to that obtained in our measurements. The consistency leads us to conclude that the slow decay component for $T_{\mathrm{c}}<T<T_{\mathrm{SC}}$ arises from FSC in $\kappa$-Br.

The magnetic torque measurements have indicated that FSC persists in both $\kappa$-Br [14] and $\kappa$-NCS [13] up to $\sim 15$ $\mathrm{K}$. In the torque measurements, the diamagnetic signal in the conducting layers is detectable in a magnetic field parallel to the layers. In this case, a large magnetic field can suppress the interlayer tunneling of Cooper pairs. As a result, the number uncertainty of the pairs may be reduced, leading to enhancement of phase fluctuation due to the uncertainty relation. Therefore, such a parallel magnetic field effect may lead to the differences in the onset temperatures between the torque and our measurements.

In the systematic ${ }^{13} \mathrm{C}-\mathrm{NMR}$ measurements, an effect of FSC has been observed only in $\kappa$-Br at $13-14 \mathrm{~K}$, but not in $\kappa$-NCS [16]. The result is qualitatively consistent with our result, though the onset temperature of the SC signal is somewhat different from ours. The difference between the onset temperatures may be due to the difficulty of determining the onset temperature in NMR measurements because even in the FSC regime, the normal metal component remains dominant, which has a proportional relation between $1 / T_{1}$ and temperature due to paramagnetism (the Korringa relation), and the FSC signal can be observed as a slight change in the slope of the temperature depen- dence. Indeed, in the report, error bars of $1 / T_{1}$ values at low magnetic fields above $13-14 \mathrm{~K}$ are relatively larger than those below $T_{\mathrm{c}}$.

FSC in $\kappa$-Br may arise owing to the phase fluctuation rather than to the conventional amplitude fluctuation. Our measurements, in principle, cannot distinguish the phase fluctuation from the amplitude fluctuation because the carrier dynamics are sensitive to the presence of the $\mathrm{SC}$ gap. However, the quantitative agreement of the onset temperature with the Nernst effect and the magnetic susceptibility measurements suggests that the gradual development in $A_{\text {slow }}$ at $T_{\mathrm{SC}}$ can have the same origin. As we discussed earlier, the Nernst signal is sensitive to the motion of vortices, indicating that the phase fluctuation is dominant above $T_{\mathrm{c}}$. Moreover, the critical behavior of diamagnetism has been analyzed in the low field limit. [15] When the temperature dependence of diamagnetism due to FSC is described by $T\left(T-T_{\mathrm{c}}\right)^{\beta}, \beta=-1$ is predicted in the case of the conventional amplitude fluctuation. The analysis yields $\beta=-2.1$, indicating that the FSC in $\kappa$-Br is more steeply developed toward $T_{\mathrm{c}}$ than FSC in the conventional superconductors. The steep growth is a notable characteristic of the phase fluctuation model.

The difference in $T_{\mathrm{SC}}$ between $\kappa$-Br and $\kappa$-NCS indicates that the emergence of FSC can be associated with electron correlation. As has been discussed in previous reports [8], because $\kappa$-Br has the smaller $t / U$ value than $\kappa$-NCS, the Cooper pairs in $\kappa$-Br tend to incur a Coulomb energy penalty, leading to reduction in the number uncertainty of the Cooper pairs $\Delta N$ [33]. From the uncertainty relation between the Cooper pair number and the phase, the phase uncertainty, $\Delta \phi$, is enhanced. The quantum fluctuation effect can lead to FSC in $\kappa$-Br.

Conclusion. - In summary, by performing polarized pump-probe spectroscopy, we found that the carrier dynamics of the SC state are characterized by polarization anisotropy for the probe with slow relaxation time in $\kappa$ NCS and $\kappa$-Br. The onset temperature of the SC response is $\sim 20 \mathrm{~K}$ in $\kappa$ - Br, which is significantly higher than $T_{\mathrm{c}}$, whereas it is just $T_{\mathrm{c}}$ in $\kappa$-NCS. From comparisons with previous studies, we conclude that the enhancement of the $\mathrm{SC}$ response above $T_{\mathrm{c}}$ can arise from FSC.

$$
* * *
$$

We would like to thank T. Mertelj, D. Mihailovic and K. Ichimura for valuable discussions. This work was supported by JSPS KAKENHI Grant No. 15K17685.

\section{REFERENCES}

[1] Xu Z. A., Ong N. P., Wang Y., Kakeshita T. and Uchida S., Nature, 406 (2000) 486. 
[2] Wang Y., Xu Z. A., Kakeshita T., Uchida S., Ono S., Ando Y. and Ong N. P., Phys. Rev. B, 64 (2001) 224519.

[3] Timusk T. and Statt B., Rep. Prog. Phys., 62 (1999) 61.

[4] Fujita K., Kim C. K., Lee I., Lee J., Hamidian M. H., Firmo I. A., Mukhopadhyay S., Eisaki H., Uchida S., Lawler M. J., Kim E.-A. and Davis J. C., Science, $\mathbf{3 4 4}$ (2014) 612.

[5] Hǘker M., Christensen N. B., Holmes A. T., Blackburn E., Forgan E. M., Liang R., Bonn D. A., Hardy W. N., Gutowski O., Zimmermann M. v., Hayden S. M. and Chang J., Phys. Rev. B, 90 (2014) 054514.

[6] Campi G., Bianconi A., Poccia N., Bianconi G., Barba L., Arrighetti G., Innocenti D., Karpinski J., Zhigadlo N. D., Kazakov S. M., Burghammer M., Zimmermann M. v., Sprung M. and Ricci A., $\mathrm{Na-}$ ture, 525 (2015) 359.

[7] Jacobs T., Katterwe S. O. and Krasnov V. M., Phys. Rev. B, 94 (2016) 220501.

[8] Nam M.-S., Ardavan A., Blundell S. J. and SChlueter J. A., Nature, 449 (2007) 584.

[9] Nam M.-S., Méziere C., Batail P., Zorina L., Simonov S. and Ardavan A., Sci. Rep., 3 (2013) 3390.

[10] Kini A. M., Geiser U., Wang H. H., Carlson K. D., Williams J. M., Kwok W. K., Vandervoort K. G., Thompson J. E. and Stupka D. L. A., Inorg. Chem., 29 (1990) 2555.

[11] Urayama H., Yamochi H., Saito G., Nozawa K., Sugano T., Kinoshita M., Sato S., Oshima K., Kawamoto A. and Tanaka J., Chem. Lett., 17 (1988) 55.

[12] Wosnitza J., Wanka S., Hagel J., Reibelt M., Schweitzer D. and Schlueter J., Synthetic Metals, 133-134 (2003) 201.

[13] Tsuchiya S., Yamada J., TAnda S., Ichimura K., Terashima T., Kurita N., Kodama K. and Uji S., Phys. Rev. B, 85 (2012) 220506.

[14] Tsuchiya S., Yamada J., Terashima T., Kurita N., Kodama K., Sugit K. and Uji S., J. Phys. Soc. Jpn., 82 (2013) 064711.

[15] Uehara T., Ito M., Taniguchi H. and Satoh K., J. Phys. Soc.Jpn., 82 (2013) 073706.

[16] Kobayashi T., Ihara Y., Saito Y. and Kawamoto A., Phys. Rev. B, 89 (2014) 165141.

[17] Li W., Zhang C., Wang X., Chakhalian J. and XiaO M., J. Magn. Magn. Mater., 376 (2015) 29.

[18] Giannetti C., Capone M., Fausti D., Fabrizio M., Parmigiani F. and Mihailovic D., Adv. Phys., 65 (2016) 58.

[19] Madan I., Kurosawa T., Toda Y., Oda M., Mertelu T. and Mihailovic D., Nat. Commun., 6 (2015) 6958.

[20] Anzai H., Delrieu J., Takasaki S., Nakatsuji S. and YAmada J., J. Cryst. Growth, 154 (1995) 145.

[21] Toda Y., Mertelu T., Naito T. and Mihailovic D., Phys. Rev. Lett., 107 (2011) 227002.

[22] Müller J., Lang M., Steglich F., Schlueter J. A., Kini A. M. and Sasaki T., Phys. Rev. B, 65 (2002) 144521.

[23] Kabanov V. V., Demsar J., Podobnik B. and Mihailovic D., Phys. Rev. B, 59 (1999) 1497.
[24] Madan I., Kurosawa T., Toda Y., Oda M., MertelJ T., Kusar P. and Mihailovic D., Scientific Reports, 4 (2014) 5656.

[25] Toda Y., Kawanokami F., Kurosawa T., Oda M., Madan I., Mertelu T., Kabanov V. V. and MiHailovic D., Phys. Rev. B, 90 (2014) 094513.

[26] Tsuchiya S., Sugawara Y., Tanda S. and Toda Y., Journal of Optics, 17 (2015) 085501.

[27] Tsuchiya S., Nakagawa K., Yamada J., Taniguchi H. and Toda Y., Phys. Rev. B, 96 (2017) 134311.

[28] Ichimura K., Suzuki K., Nomura K. and Kawamoto A., Synth. Met., 133 (2003) 213.

[29] Arai T., Ichimura K., Nomura K., Takasaki S., Yamada J., Nakatsuji S. and Anzai H., Phys. Rev. B, 63 (2001) 104518.

[30] Dressel M., Klein O., Grüner G., Carlson K. D., Wang H. H. and Williams J. M., Phys. Rev. B, 50 (1994) 13603.

[31] Mattis D. C. and Bardeen J., Phys. Rev., 111 (1958) 412.

[32] Kusar P., Kabanov V. V., Demsar J., Mertelu T., Sugai S. and Mihailovic D., Phys. Rev. Lett., 101 (2008) 227001.

[33] Doniach S., Phys. Rev. B, 24 (1981) 5063. 